\title{
A New Approach to Stochastic Reduced Order Modeling
}

Kinnar Sen ${ }^{1}$ and Urmila Diwekar ${ }^{1,2}$

\begin{abstract}
:
This short note presents a new method for stochastic reduced order (SROM) model based on BONUS reweighting scheme. An illustrative case study of IGCC power plant compares the new method with the neural network based reduced order model. The new method shows promising results.
\end{abstract}

Keywords: Reweighting scheme; BONUS algorithm; IGCC system; Reduced order model

\section{Introduction}

Reduced order modelling is becoming increasingly popular as a simpler and more economical alternative for analyzing many real-life processes. This is because such processes or systems are so complex that the mathematical models created to represent them are in turn complicated and have many dimensions. Therefore, using such models in numerical simulations and optimization to analyze the process can become an expensive and time-consuming affair because of the multiple iterations involved in running a simulation. Therein lays the benefit of reducing the order of mathematical models by employing approximations. However, this gives rise to the challenge of maintaining the accuracy of system characteristics and response. This can be overcome by being careful in deciding the approximation strategy and in importing data from the original system into the reduced order models (ROM). Thus, if we are able to retain sufficient accuracy, the ROMs obtained can be used to predict the results with substantial precision in considerably lesser amount of time. It is because of this reason that ROM has always been a topic of great interest for researchers.

Bai, Dewilde and Freund [1] have discussed the various ROM techniques used in simulating large scale linear dynamical systems such as electronic circuits and microelectromechanical systems, thus replacing methods such as RCL sub-circuits that have been traditionally applied to model VLSI circuits. Rao, Schaft, Eunen, Bakker and Jayawardhana [2] have worked on developing model order reduction using the Kron reduction of weighted Laplacian matrices. They have illustrated the application of this technique in several chemical and biochemical reaction networks governed by general enzyme kinetics. Generally, to analyze such reactions a set of stoichiometric differential equations involving a huge number of metabolites and chemical

1. Department of Industrial Engineering, University of Illinois at Chicago, Chicago, IL 60607

2. Vishwamitra Research Institute, 2714 Crystal Way, Crystal Lake, IL 60012, urmila@vricustom.org

(C) 2016. This manuscript version is made available under the Elsevier user license

http://www.elsevier.com/open-access/userlicense/1.0/ 
species have to be solved. In their work, they have reduced the number of metabolites and reactions without any major effect on the dynamics of the original reactions. Washabaugh, Amsallem, Zahr and Farhat [3] have ventured into a lesser explored area by developing a nonlinear model reduction method replacing CFD models based on the concept of local reducedorder bases. The method is executed in two phases -online and offline. The local base is developed during the offline phase and it is updated in the online phase based on the current state of the system.

In this paper, we present a new approach to reduced order modeling and applied it to analyze the process of power production in an IGCC power plant. The ROM generated by this method can be categorized as stochastic ROMs (SROMs) as it can be used for obtaining stochastic estimates such as expected value, variance of outputs. SROMs are very useful in applications such as sensor and controls. There is very little work done in the area of stochastic ROMs. Warner, Grigoriu and Wilkins [4] present a method for constructing Stochastic Reduced Order Models (SROMs) to efficiently solve random eigenvalue problems encountered in the modal analysis of structural systems with uncertain properties. They have developed an improved algorithm to construct SROMs. The algorithm is able to considerably improve the model accuracy and efficiency. They determined analytical bounds to solve random eigenvalue problems and accounted for uncertainty propagation through the mathematical model. The models thus formulated were shown to clearly outperform the classic Monte Carlo simulations in numerical examples. However, this approach requires open equation system and linear models.

Aquino et al. [5] use a Stochastic Reduced Order Model (SROM) for uncertainty quantification in systems undergoing corrosion. They demonstrated the application of SROM models for characterizing input uncertainty and propagating the uncertainty to the outputs through the mathematical model. They have developed SROMs for a random vector input $\mathrm{X}$ as a set of probable values $\mathrm{x}$ and their corresponding probabilities $\mathrm{p}$. The SROM statistics were then calculated and an optimization problem was solved to minimize the error between the SROM statistics and the actual target marginal. When it comes to computationally intensive problems like corroding systems, SROMs are more efficient than the traditional techniques such as spectral nethods, Monte Carlo techniques and stochastic calculus approaches.

1. Department of Industrial Engineering, University of Illinois at Chicago, Chicago, IL 60607

2. Vishwamitra Research Institute, 2714 Crystal Way, Crystal Lake, IL 60012, urmila@vricustom.org 
Stephen Zitney et al. [6] use ROM to replace Fluent CFD models for Gasification and Combined Cycle Combustion Units in IGCC power plant. Accurate CFD models can be developed for key units like Gasification and Combined Cycle Combustion Units. However, developing integrated flowsheets and subsequent optimization is difficult. So they developed two ROMs - Neural Networks \& Kriging and integrated them in IGCC process. The performance of the ROMs was evaluated and a $7 \%$ increase in power output was obtained by optimizing these models.

The aim of this paper is to compare Stochastic Reduced Order Models for IGCC system created using two different techniques. One of them is Neural Networks which has been fairly applied in building reduced order models. Stochastic simulations are then carried out with this ROM to obtain stochastic ROM. The second and the new approach is based on Better Optimization of Nonlinear Uncertain Systems (BONUS) reweighting approach. We present the case study of an IGCC plant where the rigorous model used for IGCC in this work is taken from the DOE/NETL Case study 8 report [7]. It has been demonstrated in this paper that the latter produces a more accurate SROM more efficiently and thus is useful in optimizing sensors and control for this system.

\section{BONUS REWEIGHTING APPROACH}

The reweighting approach is the basis of the BONUS algorithm proposed by Sahin and Diwekar (2004). BONUS uses reweighting scheme which is the basis of the SROM presented here. . Figure 1 presents the reweighting scheme in perspective. Initially we assume that our inputs are uniformly distributed between minimum and maximum value. We sample these uniformly distributed variables and propagate the sample through our rigorous model. We obtain the output distributions for this initial base distribution based on the rigorous model. Once we have these probability distributions then if the input distributions change then we do not have to sample and propagate these new distributions through the model but use reweighting scheme to obtain the corresponding output distribution. For details, please refer to Diwekar and David (2015). This reweighting approach is beneficial for eliminating the need to recreate a set of new sample points and propagating it through the rigorous model. To obtain SROM for the IGCC system, on the first iteration, a set of $\mathrm{N}_{\mathrm{s}}$ sample points uniformly distributed across a d-dimensional sample

1. Department of Industrial Engineering, University of Illinois at Chicago, Chicago, IL 60607

2. Vishwamitra Research Institute, 2714 Crystal Way, Crystal Lake, IL 60012, urmila@vricustom.org 
space are used to perform $\mathrm{N}_{\mathrm{s}}$ simulation replications of the IGCC process (i.e., at various operating points) rigorous model. Let $\mathrm{f}_{0}\left(\mathrm{x}_{\mathrm{i}}\right)$ and $\mathrm{F}_{0}\left(\mathrm{x}_{\mathrm{i}}\right)$ be the probability density function (pdf) and cumulative distribution function (cdf) associated with the base input distribution for the input variable $\mathrm{x}_{\mathrm{i}}, \mathrm{i}=1,2, \ldots, \mathrm{S}_{\mathrm{in}}$, respectively. Following the simulation of the IGCC process at iteration $\mathrm{k}=1$, let $\mathrm{f}_{0}\left(\mathrm{y}_{\mathrm{j}}\right)$ and $\mathrm{F}_{0}\left(\mathrm{y}_{\mathrm{j}}\right)$ be the base probability density function (pdf) and cumulative probability density function (cdf) associated with the intermediate and output variable $\mathrm{y}_{\mathrm{j}}, \mathrm{j}=$ $1,2, \ldots, \mathrm{S}_{\text {out }}$, respectively.

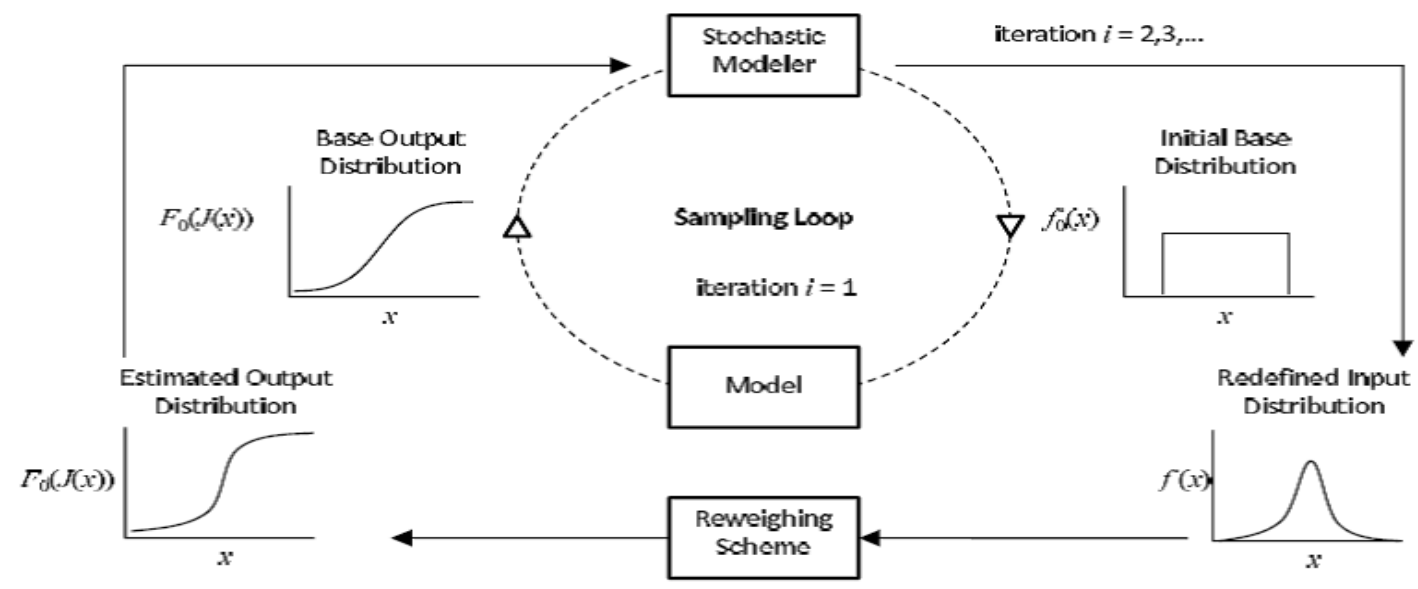

Figure 1- The reweighting approach

Next, consider when the input distribution is redefined, such as when a sensor is placed at the location of this input variable. The redefined distribution, $\mathrm{f}_{\mathrm{k}}\left(\mathrm{x}_{\mathrm{i}}\right)$, is used to create a set of weights,

$$
W_{k}\left(x_{i}\right)=\frac{f_{k}\left(x_{i}\right)}{f_{0}\left(x_{i}\right)} \ldots i=1,2, \ldots, S_{i n}
$$

which gives the likelihood ratio between the redefined and base distributions. Given that the input variables act independently, these weights are used to construct the resulting distribution for the downstream intermediate and output variables by multiplying the associated weights $\mathrm{W}$ $\left(\mathrm{x}_{\mathrm{i}}\right)$ with the base distribution $\mathrm{f}_{0}\left(\mathrm{y}_{\mathrm{j}}\right)$

1. Department of Industrial Engineering, University of Illinois at Chicago, Chicago, IL 60607

2. Vishwamitra Research Institute, 2714 Crystal Way, Crystal Lake, IL 60012, urmila@vricustom.org 


$$
f_{k}\left(y_{i}\right)=f_{0}\left(y_{i}\right) \prod_{i=1}^{s_{\text {in }}}\left(1+\gamma_{i j}\left(W_{k}\left(x_{i}\right)-1\right) \ldots j=1,2, \ldots S_{\text {out }}\right.
$$

Where $\gamma_{i j}=1$ if variable $y_{j}$ is downstream of $x_{i}$ and $\gamma_{i j}=0$ if variable $y_{j}$ is not downstream of $x_{i}$. The above distribution $\mathrm{f}_{\mathrm{k}}\left(\mathrm{y}_{\mathrm{j}}\right)$ is then normalized using

$$
f_{k}\left(y_{i}\right)=\frac{f_{k}\left(y_{i}\right)}{\sum_{n=1}^{N_{s}} f_{k}\left(y_{n}\left(y_{n+1}-y_{n-1}\right) / 2\right)}
$$

Although, the reweighting equations appear to be linear, they are linear in probability space and can easily be used for nonlinear models and is shown applicable to IGCC system below.

This reweighting approach is thus used to construct the resulting change in distributions of corresponding downstream variables by eliminating the need to regenerate a new set of Ns sample points through simulation of the IGCC process. The number of sample points depends on the number of input variables. Here we have used 800 sample points with 8 input variables. There are large number of intermediate and output variables in this flowsheet. The reweighting approach explained above can be used as a SROM for the IGCC process.

\section{METHODOLOGY}

A set of Ns sample points for each of the input parameters was generated using Hammersley Sampling Sequence (HSS) which is a low discrepancy sampling method [11] This sampling technique is preferred over the commonly used Monte Carlo simulation because it can provide a more uniform distribution and better coverage of any d-dimensional sample space using lesser number of sample points. The input variables were assumed to follow a uniform distribution and the samples were generated accordingly.

1. Department of Industrial Engineering, University of Illinois at Chicago, Chicago, IL 60607

2. Vishwamitra Research Institute, 2714 Crystal Way, Crystal Lake, IL 60012, urmila@vricustom.org 
Then, the entire IGCC plant modeled in ASPEN is simulated $\mathrm{N}_{\mathrm{s}}$ times, once per input operating condition, to generate a corresponding vector of points for Sout intermediate and output process variables. The input and output data sets thus obtained from our base data which will later form our basis of comparison.

This considerably large amount of data acquired from $\mathrm{N}_{\mathrm{s}}$ samples is used to develop a Neural Network (NN), which is our first Reduced Order Model to predict the behavior of the IGCC plant. $70 \%$ of the data is used in the training phase whereby both input and output data sets are made available to the NN so that it can follow the trends in the data to "learn" how the IGCC plant functions. Then, the next $15 \%$ of the data is used for validation and finally the predictive power of the $\mathrm{NN}$ is tested using the last $15 \%$ of the data.

The trained Neural Network is now ready to be used on any new, unrelated data set. To test the efficacy of the $\mathrm{NN}$, we generated a new input data set consisting of $\mathrm{N}_{\mathrm{s}}$ sample points. However, this time, instead of uniform distribution, we considered normal distribution (and then lognormal distribution) for all the input variables.

Thereafter, we used the reweighting approach to develop our second ROM. The input data set following uniform distribution was considered as our base and that generated by HSS as following normal distribution (and log-normal distribution) was used for calculating weights. Reweighting was done with the help of the base output generated by ASPEN simulation and used in calculating the output variables in case the probability distribution of the input variables changed to normal (and log-normal).

The ASPEN simulation was run again, this time with the input variables following normal (and log-normal) distribution to check the accuracy of the two SROMs. These simulations are used for checking the accuracy of the two SROMs.

The values of several outputs such as gas turbine power production, steam turbine power production, overall thermal efficiency etc. predicted by the two ROMs were compared to see which is closer to the output values predicted by the simulation model created in ASPEN.

\section{RESULTS}

In this section, we are conducting a case study of the IGCC power plant wherein a uniform 8dimensional sample space of potential operating conditions for the input variables is generated by creating a set of sample points across $+/-10 \%$ of the nominal conditions using the

1. Department of Industrial Engineering, University of Illinois at Chicago, Chicago, IL 60607

2. Vishwamitra Research Institute, 2714 Crystal Way, Crystal Lake, IL 60012, urmila@vricustom.org 
Hammersley sequence sampling method. The nominal values of the input process variables are shown below in table 1 .

\begin{tabular}{|l|l|l|l|}
\hline Input & Description & Nominal & Units \\
\hline 1 & Oxygen flow rate to ASU & 157392 & $\mathrm{~kg} / \mathrm{hr}$ \\
\hline 2 & Coal slurry flow rate to gasifier & 57288.7 & $\mathrm{~kg} / \mathrm{hr}$ \\
\hline 3 & Air flow rate to gas turbine compressor & 2962684 & $\mathrm{~kg} / \mathrm{hr}$ \\
\hline 4 & Recycled HRSG steam temperature & 141.111 & $\mathrm{C}$ \\
\hline 5 & Recycled HRSG steam pressure & 526070 & $\mathrm{~Pa}$ \\
\hline 6 & Recycled HRSG water temperature & 96.1111 & $\mathrm{C}$ \\
\hline 7 & Gasifier temperature & 1371.111 & $\mathrm{C}$ \\
\hline 8 & Gasifier pressure & 2806166 & $\mathrm{~Pa}$ \\
\hline
\end{tabular}

Table 1 - Input Process Variables

Now, for each set of operating conditions, the corresponding intermediate \& output variable conditions are generated using a steady-state model developed in the ASPEN Plus simulation environment. A distribution function is constructed from these sets of sample points using kernel density estimation technique [12] which serves as the base distribution in the reweighting scheme. Moreover, the input and output data set obtained using ASPEN plus simulation environment is required to train and generate the Neural Network.

In a similar way, a Normal and then a Log-Normal 8-dimensional sample space of potential operating conditions for the input variables are generated by creating a set of sample points across $+/-10 \%$ of the nominal conditions using the Hammersley sequence sampling method.

Again, for each set of operating conditions, the corresponding intermediate and output variable conditions are generated using a steady-state model developed in the ASPEN Plus simulation environment.

The results obtained from ASPEN simulation environment were used as yardstick to compare the accuracy of the predictions made by the two Reduced Order Models - Neural Network \& the reweighting approach.

The output obtained from the ASPEN simulation environment as well as two ROMS, viz., NN and reweighting, are tabulated below in Table 2. After comparing the predictions made by the two ROMs to the output generated by ASPEN plus simulation environment we observe that, in most of the cases, the values predicted by the reweighting approach are closer to those predicted by ASPEN and are hence more accurate. The results shown in bold in Table 2 are the more accurate ones.

1. Department of Industrial Engineering, University of Illinois at Chicago, Chicago, IL 60607

2. Vishwamitra Research Institute, 2714 Crystal Way, Crystal Lake, IL 60012, urmila@vricustom.org 


\begin{tabular}{|c|c|c|c|c|c|c|c|}
\hline \multirow{2}{*}{\multicolumn{2}{|c|}{$\begin{array}{l}\text { Expected Value of the } \\
\text { Variable }\end{array}$}} & \multicolumn{3}{|c|}{ Normal Distribution } & \multicolumn{3}{|c|}{ Log-Normal Distribution } \\
\hline & & $\begin{array}{l}\text { Aspen } \\
\text { Simulation }\end{array}$ & $\begin{array}{l}\text { Neural } \\
\text { Network }\end{array}$ & $\begin{array}{l}\text { Reweightin } \\
\text { g }\end{array}$ & $\begin{array}{l}\text { Aspen } \\
\text { Simulation }\end{array}$ & $\begin{array}{l}\text { Neural } \\
\text { Network }\end{array}$ & $\begin{array}{l}\text { Reweight } \\
\text { ing }\end{array}$ \\
\hline \multirow{6}{*}{$\begin{array}{l}\text { IGCC } \\
\text { power } \\
\text { production }\end{array}$} & $\begin{array}{l}\text { Gas turbine } \\
\text { power } \\
\text { production } \\
\text { (MWE) }\end{array}$ & 425.219 & 486.19 & 440 & 423.74 & 334.01 & 438.21 \\
\hline & $\begin{array}{l}\text { Steam turbine } \\
\text { power } \\
\text { production } \\
\text { (MWE) }\end{array}$ & 252.44 & 253.63 & 260 & 250.38 & 250.19 & 259.83 \\
\hline & $\begin{array}{l}\text { Misc. power } \\
\text { production } \\
(\mathrm{MWE})\end{array}$ & -67.43 & -61.63 & -69.8 & -67.03 & -61.43 & -69.51 \\
\hline & $\begin{array}{l}\text { Auxiliary } \\
\text { power } \\
\text { production } \\
\text { (MWE) }\end{array}$ & 18.31 & 20.86 & 18.93 & 18.21 & 15.95 & 18.85 \\
\hline & $\begin{array}{l}\text { Total plant } \\
\text { power } \\
\text { production } \\
\text { (MWE) }\end{array}$ & 591.99 & 591.03 & 612.17 & 588.89 & 582.57 & 609.67 \\
\hline & $\begin{array}{l}\text { Plant Thermal } \\
\text { Efficiency }\end{array}$ & 40.71 & 39.7 & 40.83 & 40.69 & 41.48 & 40.81 \\
\hline
\end{tabular}

\section{Table 2 - Output Process Variables}

It should be noted here that the results generated by the Neural Network change, albeit slightly, with each run. This is mainly due to random weight initialization and random data division during the training and validation phases. This randomness is essential to ensure that the neural network learns the actual patterns in the data without giving too much credence to the particular data set used for training.

\section{CPU TIME COMPARISON}

1. Department of Industrial Engineering, University of Illinois at Chicago, Chicago, IL 60607

2. Vishwamitra Research Institute, 2714 Crystal Way, Crystal Lake, IL 60012, urmila@vricustom.org 
The CPU time for the two SROMs as well as the original ASPEN model is presented in Table 3. It can be clearly seen that the time required for running the ASPEN simulation is really high as compared to the other methods. This demonstrates the advantage of using reduced order modelling.

Moreover, the time required to solve the problem using the reweighting approach is about 10-20 times lesser than that of the Neural Network SROM. This is because, unlike NN, the reweighting approach does not need to spend time on training and validation and is thus much faster.

As the magnitude of the problem increases, it is expected that the CPU times for NN will increase at a greater rate than the reweighting approach. This is because the NN will have to spend more time while processing increased volumes of data for training and validation.

\begin{tabular}{|l|l|}
\hline \multicolumn{2}{|c|}{ NORMAL DISTRIBUTION } \\
\hline ASPEN Simulation & 9 Hours \\
\hline Neural-Network & $5-12$ seconds \\
\hline Reweighting & 0.4219 second \\
\hline \multicolumn{2}{|c|}{ LOG-NORMAL DISTRIBUTION } \\
\hline ASPEN Simulation & 18 Hours \\
\hline Neural-Network & $5-12$ seconds \\
\hline Bonus & 0.5313 second \\
\hline
\end{tabular}

\section{Table 3: CPU Time comparison}

\section{CONCLUSION}

In this paper, we have proposed a simple method to compare the predictions made by two Reduced Order Models (ROMs) viz. - Neural Networks and reweighting approach to generate the output variables in an IGCC power plant when the sample space of Uniformly distributed potential operating conditions for the input process variables are changed to Normal \& LogNormal distributions. Reweighting approach is demonstrated to make predictions which are more reliable than that made by the Neural Network. Hence this paper recommends the use of reweighting approach over Neural Networks as a Reduced Order Model for prediction of output variables in an IGCC power plant when the input variables are modified.

Acknowledgement: This work was funded by DOE NETL under grant \#: DE-FE0011227 REFERENCES

1. Department of Industrial Engineering, University of Illinois at Chicago, Chicago, IL 60607

2. Vishwamitra Research Institute, 2714 Crystal Way, Crystal Lake, IL 60012, urmila@vricustom.org 
[1] Bai Z, Dewilde PM, Freund RW. Reduced-Order Modeling. Handbook of Numerical Analysis Vol. XIII, Numerical Methods in Electromagnetics, W. Schilders and E.J.W. ter Maten, eds. pp. 825-895, 2005.

[2] Rao S., van der Schaft AJ, van Eunen K, Bakker BM, Jayawardhana B. Model-order reduction of biochemical reaction networks. BMC Systems Biology, 8, [52]. 10.1186/1752-0509-8-52.

[7] US Department of Energy National Energy Technology Laboratory. EPA study - case 8: IGCC. Technical report DOE/NETL-401/042606, November 2006.

[8] Martelli E, Kreutz T, Carbo M, Consonni S, Jansen D. Shell coal IGCCS with carbon capture: conventional gas quench vs. innovative configurations. Appl Energy 2011;88:3978-89

[9] Giuffrida A, Romano MC, Lozza GG. Thermodynamic assessment of IGCC power plants with hot fuel gas desulfurization. Appl Energy 2010;87:3374-83.

[10] Giuffrida A, Romano MC, Lozza G. Thermodynamic analysis of air-blown gasification for IGCC applications. Appl Energy 2011;88:3949-58.

[11] Wang R, Diwekar U, Catherine E, Padro G. Efficient sampling techniques for uncertainties is risk analysis. Wiley Interscience 2004 : DOI 10.1002/ep.10020.

[12] Silverman BW, Density estimation for statistics and data analysis. Monographs on Statistics and Applied Probability, London: Chapman and Hall, 1986

1. Department of Industrial Engineering, University of Illinois at Chicago, Chicago, IL 60607

2. Vishwamitra Research Institute, 2714 Crystal Way, Crystal Lake, IL 60012, urmila@vricustom.org 